\title{
Beta-interferon, retinoids and tamoxifen in metastatic breast cancer: Long-term follow-up of a phase II study
}

\author{
FRANCESCO RECCHIA ${ }^{1,2}$, GIGLIOLA SICA ${ }^{3}$, GIAMPIERO CANDELORO ${ }^{1}$, STEFANO NECOZIONE $^{4}$, \\ ROBERTA BISEGNA ${ }^{1}$, MASSIMO BRATTA ${ }^{2}$ and SILVIO REA ${ }^{2,5}$ \\ ${ }^{1}$ Ospedale Civile di Avezzano, Unità Operativa di Oncologia; ${ }^{2}$ Fondazione 'Carlo Ferri', Monterotondo; \\ ${ }^{3}$ Istituto di Istologia ed Embriologia, Università Cattolica del Sacro Cuore, Roma; ${ }^{4}$ Epidemiologia Clinica, \\ Università degli Studi dell'Aquila; ${ }^{5}$ Chirurgia Oncologica, Università degli Studi dell'Aquila, L'Aquila, Italy
}

Received October 31, 2008; Accepted December 12, 2008

DOI: $10.3892 /$ or_00000317

\begin{abstract}
Based on a series of in vitro data, including the additive and/or synergistic antiproliferative effect of interferon and tamoxifen on breast cancer cell lines, and on clinical reports, we designed a pilot phase II study to test the activity and toxicity of simultaneous administration of $\beta$-interferon (B-IFN), retinoids (R) and tamoxifen (TAM) as a salvage therapy in a group of patients with metastatic breast cancer (MBC). Herein we describe the outcome of this cohort of patients after a median follow-up of 150 months. Sixty-five stage IV breast cancer patients, 13 pre-treated with hormones, 38 with chemotherapy and 15 with both, received, as a salvage therapy, TAM, B-IFN and R. Among 65 evaluable patients, 36 achieved a clinical response (55.5\%) (95\% c.i. 42-67.7\%). Toxicity was moderate and mainly hepatic. Median progression-free and overall survival, which did not show any statistically significant difference in patients with different estrogen and progesterone receptor content, were 43 months and 47.9 months, respectively. In conclusion, the study shows that long-term treatment with TAM, B-IFN and R in MBC is feasible, has moderate toxicity and seems to give a long-term benefit, irrespective of the receptorial status.
\end{abstract}

\section{Introduction}

The primary purpose of treatment of metastatic breast cancer (MBC) is palliative, in order to ameliorate quality, and perhaps also duration of life without any realistic hope for cure (1). Tamoxifen (TAM), a potent antiestrogen, has been the most widely accepted form of endocrine therapy for advanced disease (2). In patients (Pts) with estrogen and progesterone receptor (ER, $\mathrm{PgR})$-positive tumors, a response rate in the range of $50-60 \%$, and a median progression-free survival of

Correspondence to: Dr F. Recchia, Oncologia, Ospedale Civile di Avezzano, via G. Di Vittorio s.n.c., 67051 Avezzano, AQ, Italy E-mail: frecchia1946@libero.it

Key words: metastatic breast cancer, B-interferon, retinoids, tamoxifen $\sim 7$ months may be expected (2). In recent years, the introduction of aromatase inhibitors (AIs) has improved the clinical outcome of breast cancer endocrine therapy. In randomized trials, anastrazole (3), letrozole (4) and examestane (5) have shown a better progression-free survival (PFS) compared with TAM in MBC. However, even in responders, invariably resistance to hormones will develop and the disease relapses (6). With the aim of improving response of MBC to hormonal therapy, in a previous study we combined TAM with $B$ interferon (B-IFN) and retinoids (R) (7). Prompted by the encouraging results obtained with the clinical study, we verified, in vitro, the growth inhibitory action of $\mathrm{R}$ alone or combined with B-IFN on both ER-positive and -negative breast cancer cell lines. Our results clearly indicated that $\mathrm{R}$ inhibited the growth of both $\mathrm{ER}^{+}$and ER-human breast cancer cell lines and that the combination of $\mathrm{R}$ with B-IFN induced an antiproliferative effect more pronounced than that produced by each of the two single agents, being the inhibitory effect synergistic in estrogen-sensitive cells (8).

Other authors obtained similar results with different models (9). The rationale for using interferons was due to the capacity that these agents have to inhibit the growth of both ER-positive and ER-negative breast cancer cell lines (10), being B-IFN more active (11), and to sensitize estrogen-responsive cells to the antiproliferative action of TAM (12). The observed increase in cell growth inhibition could be due to the enhancement of ER which may allow an increased anti-estrogenreceptor interaction (13). Retinoids as well as TAM induce the secretion of transforming growth factor- $\beta$ that inhibits the growth of most epithelial cells (14). In some breast cancer cells, they have been shown to enhance the growth inhibitory action of various anti-estrogens (15). They are also able to increase ER in both TAM-sensitive and resistant sublines of breast cancer cell line MCF-7 and to enhance PgR level in T47D cells in which the expression of these receptors is independent of estrogen control $(16,17)$.

Patients with advanced breast cancer have been reported to have a variety of functional abnormalities of the immune system, which may differ in magnitude from one subject to another one and which may be related to the extent of disease (18). As a result, the immuno-inhibitory influence of the tumor might extend far beyond the tumor microenvironment and become a systemic effect, especially in Pts with advanced and 
metastatic disease. Both $\beta$-IFN and R may have an important role in improving the immune function. It has been shown that B-IFN upregulates the expression of breast cancer tumor antigens, and $\mathrm{R}$ increase the percentage of peripheral blood lymphoid cells expressing surface markers for T-helper cells $(19,20)$.

Herein we describe a further follow-up of a phase II pilot study, previously published (7), that included B-IFN, R and TAM as a salvage treatment of MBC.

\section{Patients and methods}

Patients. In the present study, we report on 65 Pts, affected by metastatic inoperable, histologically proven advanced breast cancer, with evidence of progressive disease. Details on Pts recruitment and entry criteria have been previously described (7). Forty-nine Pts were entered onto the protocol, from March 1988 to July 1992. Additional 16 Pts were recruited from July 1992 to December 1994. The extension of Pts accrual increased the median follow-up time to 150 months. Written informed consent, approved by the Institutional Review Board, was obtained from each patient.

Treatment plan. The dose of TAM, given until progression, was established at $30 \mathrm{mg}$ /day because 28 patients had been pretreated with $20 \mathrm{mg}$ and had progressed and because there have been studies suggesting that the higher dosage of TAM might have a better antitumor effect $(21,22)$. B-IFN was administered at the dose of $1 \times 10^{6} \mathrm{IU} / \mathrm{m}^{2}$ subcutaneously 3 times a week and retynil palmitate, 15,000 IU orally twice per day. During the second year, and thereafter, in order to decrease toxicity, therapy was continued in responders, with B-IFN $1 \times 10^{6} \mathrm{IU} / \mathrm{m}^{2}$ subcutaneously once a week and retynil palmitate, 15,000 IU orally once a day for 5 days/week, 2 weeks each month until progression. When fever and constitutional symptoms occurred, $\beta$-IFN administration was preceded by $500 \mathrm{mg}$ acetaminophen. If hematological toxicity occurred, administration of therapy was delayed until platelet counts were $\geq 100 \times 10^{9} / 1$ and absolute granulocytes were $\geq 1 \times 10^{9} / 1$. If gastrointestinal toxicity occurred, the administration of $B$-IFN and $\mathrm{R}$ was delayed until optimal dose could be tolerated. All women underwent an outpatient treatment and received therapy at home. At disease progression with TAM, an aromatase inhibitor was administered, followed by chemotherapy.

Statistical considerations. The study was designed to test the hypothesis that the hormonal therapy adopted might be effective in the treatment of MBC Pts who had been pretreated with hormones and had progressed. Simon's optimal two-stage design was used (23). According to this design, the first stage required a confirmed response in at least 8 responders out of $24 \mathrm{Pts}$. This criterion would rule out an undesirably low response probability of $0.30(\mathrm{P} 0)$, in favor of a desirable response probability of $0.50(\mathrm{P} 1)$, with a $5 \%$ probability of accepting a poor agent $(\alpha=0.05)$ and a $10 \%$ probability of rejecting a good agent $(\beta=0.1)$ before proceeding to the second stage. In the second stage, if 24 or more Pts achieved a confirmed response on a total of 63 assessable Pts, than the primary end-point would have been met. The time to progression was defined as the time between
Table I. Characteristics of patients.

\begin{tabular}{|c|c|c|}
\hline Characteristics & No. & $\%$ \\
\hline No. of patients & 65 & 100 \\
\hline \multicolumn{3}{|l|}{ Age, years } \\
\hline Median & 62 & \\
\hline Range & $28-75$ & \\
\hline \multicolumn{3}{|c|}{ Performance status (ECOG) } \\
\hline $0-1$ & 41 & 63 \\
\hline 2 & 22 & 34 \\
\hline 3 & 2 & 3 \\
\hline \multicolumn{3}{|l|}{ Disease-free interval } \\
\hline Median 28 months & 47 & 72 \\
\hline Stage IV at diagnosis & 18 & 28 \\
\hline \multicolumn{3}{|l|}{ Estrogen receptors } \\
\hline $\mathrm{ER}^{+}$ & 30 & 46 \\
\hline $\mathrm{ER}^{-}$ & 11 & 17 \\
\hline Unknown & 24 & 37 \\
\hline \multicolumn{3}{|l|}{ Progesterone receptors } \\
\hline $\mathrm{PgR}^{+}$ & 20 & 31 \\
\hline $\mathrm{PgR}^{-}$ & 16 & 25 \\
\hline Unknown & 29 & 44 \\
\hline \multicolumn{3}{|l|}{ Previous treatment } \\
\hline Surgery & 64 & 98 \\
\hline Chemotherapy & 53 & 82 \\
\hline Hormonotherapy & 28 & 43 \\
\hline Radiotherapy & 35 & 54 \\
\hline \multicolumn{3}{|l|}{ Menopausal status } \\
\hline Premenopause & 7 & 11 \\
\hline Postmenopause & 58 & 89 \\
\hline \multicolumn{3}{|l|}{ Dominant site of disease } \\
\hline Viscera & 25 & 38 \\
\hline Bone & 27 & 42 \\
\hline Soft tissue & 13 & 20 \\
\hline
\end{tabular}

the start of therapy and any relapse or the appearance of a second primary cancer or death, whichever occurred first. Overall survival (OS) was measured from study entry to death, or study entry to December 2007, for censored Pts. Statistical analysis was performed with SAS statistical software (version 9.1, 2003 SAS Institute Inc., Cary, NC), PFS and OS were determined using the Kaplan-Meier method (24). All comparisons of Pts characteristics, response rates and toxicity profiles were performed using Pearsons $\chi^{2}$ contingency table analysis. Analysis of data was performed in February 2008.

\section{Results}

All Pts had symptoms from their disease. The median age was 62 years (range: 28-75) with seven pre-menopausal and 
Table II. Toxicity.

\begin{tabular}{lcccc}
\hline & \multicolumn{4}{c}{ (WHO grade) } \\
\cline { 2 - 5 } & 0 & 1 & 2 & Total \\
& No. $(\%)$ & No. $(\%)$ & No. $(\%)$ & No. $(\%)$ \\
\hline Hematologic & & & & \\
$\quad$ Leucopenia & $59(91)$ & $6(9)$ & $0(0)$ & $65(100)$ \\
Neutropenia & $59(91)$ & $6(9)$ & $0(0)$ & $65(100)$ \\
Thrombocytopenia & $57(88)$ & $8(12)$ & $0(0)$ & $65(100)$ \\
Anemia & $62(95)$ & $3(5)$ & $0(0)$ & $65(100)$ \\
Gastrointestinal & & & & \\
Hepatic & & & & \\
Diarrhea & $54(83)$ & $10(15)$ & $1(2)$ & $65(100)$ \\
Triglycerides & $61(94)$ & $4(6)$ & $0(0)$ & $65(100)$ \\
Cutaneous & $57(88)$ & $8(12)$ & $0(0)$ & $65(100)$ \\
Fever & $54(83)$ & $8(12)$ & $3(5)$ & $65(100)$ \\
Autoimmune reactions & $55(85)$ & $10(15)$ & $0(0)$ & $65(100)$ \\
\hline
\end{tabular}

58 post-menopausal women. Median follow-up was 150 months. Median disease-free interval was 28 months for 47 Pts, while 18 Pts had stage IV disease at diagnosis. Initial stage of Pts at the moment of diagnosis was: stage I, 10 Pts; stage II 19 Pts; and stage III 18 Pts. Three Pts had bilateral breast cancer. ER and PgR, determined by immunohistochemical assay, were strongly positive ( $>50 \%$ of cells stained) in 30 and 20 Pts, respectively; negative in 11 and 16, and unknown in 24 and 29 Pts, respectively. Primary treatment had been modified radical mastectomy for 46 Pts; segmental mastectomies for $18 \mathrm{Pts}$; and radiotherapy for one patient. Twelve Pts (18\%) had received adjuvant chemotherapy. Twenty-eight Pts (43\%) had been pre-treated with hormones, 18 with TAM alone and 10 with TAM plus medroxyprogesterone acetate and aminogluthetimide. Five Pts had responses for a median time of 10 months, four had disease stabilization; all Pts receiving TAM had progressed. Forty-seven Pts (72\%) had received one or two lines of chemotherapy for metastatic disease. Fifteen had been treated with both hormones and chemotherapy. Pts characteristics are listed in Table I.

Activity. Among 65 evaluable Pts, objective overall remission was observed in 36 women (55.5\%) (95\% c.i. $42.5 \%-67.7 \%)$ (Table III). Fourteen Pts achieved a complete response (21.5\%), (95\% c.i. $12.3 \%-33.5 \%)$; 22 a partial response response (34\%), (95\% c.i. $22-5 \%-46.6 \%)$. Disease stability was observed in 14 Pts $(21.5 \%)$, (95\% c.i. $12.3 \%-33.5 \%)$, and progression in 15 Pts $(23 \%)$, (95\% c.i. $13.5 \%-35.2 \%)$. Autopsy was performed in two Pts; pathology confirmed the complete response in brain and liver. Median PFS and OS were 43 and 47.9 months, respectively. There was no statistically significant difference in PFS and OS, between patients with high, low or unknown ER content (data not shown). Similarly, there was no statistically significant difference in PFS $(\mathrm{P}=0.76)$ and $\mathrm{OS}$

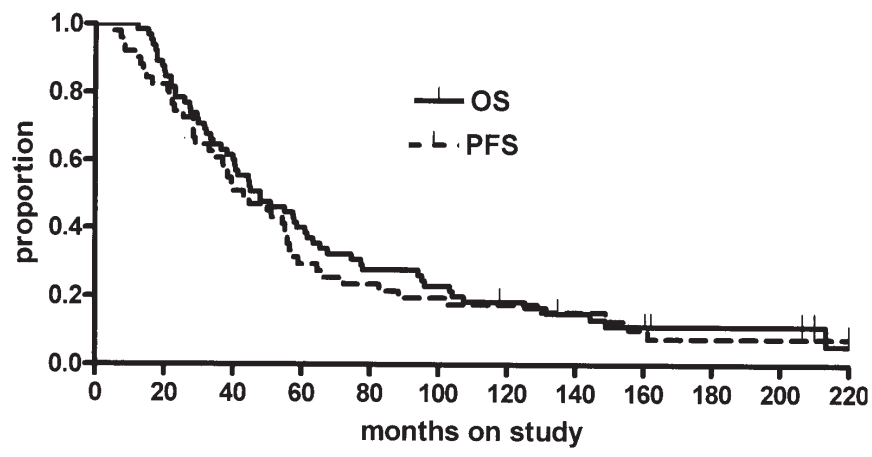

Figure 1. Progression-free survival (PFS). Events 60: (92.3\%), censored 5: $7.7 \%$, median PFS: 43 months. Overall survival (OS). Events 58: 89.2\%, censored 7: $10.8 \%$, median OS: 47.9 months.

Table III. Response to therapy.

\begin{tabular}{llc}
\hline & No. $(\%)$ & $95 \%$ CI: \\
\hline CR & $14(21.5)$ & $12.3-33.5 \%$ \\
PR & $22(34.0)$ & $22.5-46.6 \%$ \\
SD & $14(21.5)$ & $12.3-33.5 \%$ \\
PD & $15(23.0)$ & $13.5-35.2 \%$ \\
R.R. & $36(55.5)$ & $42.5-67.7 \%$ \\
\hline
\end{tabular}

No., number of patients; CR, complete response; PR, partial response; $\mathrm{SD}$, stable disease; $\mathrm{PD}$, progressive disease; $\mathrm{CI}$ : confidence intervals.

$(\mathrm{P}=0.68)$ between patients pretreated with hormonal therapy or chemotherapy. After a median follow-up of 150 months, 7 Pts $(10.8 \%)$ were alive. Responses were obtained in 18 Pts who had received chemotherapy, in 10 Pts who had progressed with TAM and in 8 Pts who had received TAM plus medroxyprogesterone acetate and aminogluthetimide. Sites of response were viscera in 16 Pts, bone in 11 Pts and soft tissue in 9 Pts. Responses in bone and soft tissue were evenly distributed, with six Pts in each group. Progressions occurred with pulmonary carcinomatous lymphangitis in 18 Pts; 19 Pts died with cerebral metastases and 21 with disseminated bone disease and malignant hypercalcemia.

Serial biopsies were performed on cutaneous lesions in four Pts; ER content increased, with respect to the previous determinations, by 20,20,30 and 100\%, respectively. PgR showed no significant variation in three Pts, while in one patient they became positive (40\%), being negative before entering the trial. The receptors became negative in one of these Pts when she failed, after six months of a partial response. A statistically significant improvement was observed in $\mathrm{CD}^{+} / \mathrm{CD}^{+}$ratio in the $46 \mathrm{Pts}$ who had shown a clinical benefit. One and three years later, the ratio increased by $16 \%$ and $23 \%$, respectively. Twenty-five Pts who had relapsed received a salvage chemotherapy with carboplatin combination with a good tolerance. Twelve Pts (18\%) with $\mathrm{ER}^{+}$tumors developed controlateral breast cancer during the follow-up time. 
Toxicity. Toxicity of therapy was acceptable (Table II) and compliance was optimal. Initially, low grade fever was observed in $11 \%$ of Pts, that was avoided, afterwards, administering $\beta$-IFN at night and preceding it with $500 \mathrm{mg}$ acetaminophen. Grade 1 skin toxicity occurred in eight Pts $(12 \%)$. Manifestations of autoimmune reactions, such as thyroiditis and reacutization of psoriasis, were observed in $15 \%$ of Pts. Elevation of triglycerides (twice baseline value) was observed in $8 \mathrm{Pts}$. Hepatic toxicity (low grade abnormality of hepatic sonogram and liver enzymes elevation 3 times baseline value) was observed in 11 Pts (17\%). Liver biopsy, performed in two, showed fatty infiltration of $30 \%$ and $60 \%$ of hepatocytes, respectively, without abnormalities of porto-biliary space, after 23 and 13 months of therapy, respectively. Both Pts indulged in alcohol consumption. Retinoids were discontinued with no further problem. In order to avoid excessive liver toxicity, retynyl palmitate, on the second year of therapy, was given 5 days/week, two weeks each month.

\section{Discussion}

In recent years, outstanding progress has been made in the treatment of MBC, with the introduction of new chemotherapeutic agents, hormones and biological agents. However, the prognosis of MBC remains poor. For almost two decades, TAM has been considered the first line hormonal treatment of advanced breast cancer in post-menopausal Pts. Premenopausal Pts, with an endocrine responsive disease, have also a benefit from TAM after adjuvant chemotherapy (25). The preminence of TAM has been challenged by the AIs, anastrazole, letrozole and examestane. All three AIs markedly suppress plasma estrogen levels via inhibition of the peripheral conversion of androgens to estrogens. In randomized trials, they showed a better clinical outcome with respect to TAM in MBC (3-5). Nevertheless, a pooled analysis of published randomized trials of first line therapy with AIs versus TAM, in hormone-responsive MBC, showed an advantage of treatment with AIs in terms of PFS and clinical benefit but not of OS or objective response (26).

When the present study was initially started, the options for Pts with hormone-responsive MBC progressing on TAM were limited to progestins and aminogluthetimide. For this reason, we tried to find an approach that could improve the efficacy of TAM. Our aim was to render breast cancer cells more susceptible to hormonal therapy and to improve the immune function with the use of B-IFN and R. Sica et al demonstrated that B-IFN may induce the increase in ER in mammary cancer cell lines and, in agreement with other authors, they showed that B-IFN treatment induce increase in both ER and PgR in cutaneous metastases of breast cancer patients $(27,28)$. The receptor increase may be potentially linked to an enhanced hormone-sensitivity.

Furthermore, $\mathrm{R}$ have been reported to induce a series of effects on breast cancer cell lines. Besides the antiproliferative action, they have been shown to promote apoptosis in both $\mathrm{ER}^{+}$and $\mathrm{ER}^{-}$models $(15,29)$. Products of retinoic acid metabolism blocking agents induce breast cancer cell differentiation (increased expression in cytokeratin and ER) and inhibit breast cancer cell growth both in vitro and in vivo (30). Moreover, it has been demonstrated that a novel retinoic acid metabolism blocking agent may reverse the resistance that develops with prolonged exposure to AIs (31). Finally, R stimulate the expression of IFN-regulated genes in IFN-resistant cells, wich suggests that combination treatment with $\mathrm{R}$ and IFNs may increase IFN-stimulated gene expression in IFNresistant tumors, leading to augmented antitumor effects (32).

One of the reasons of failure of cancer therapy is due to the fact that the immune system is compromized in Pts with advanced breast cancer (18). Such a dysfunction may appear early in the course of disease and may worsen after cancer therapy (33). B-IFN and R might have a key role in the functional improvement of cell-mediated immunity. Interferon has the capacity to enhance the tumor-associated antigens on a spectrum of freshly isolated human adenocarcinoma cells (34). Additionally, an important action of R on the immune system has been recently focused; the facilitation of the differentiation of immature myeloid suppressor cells $\left(\mathrm{Gr}-1^{+}\right.$ $\mathrm{CD} 115^{+}$), which are responsible for the development of tumorinduced T-cell anergy in tumor-bearing hosts (35), with an improvement of the immune response $(36,37)$.

In our study, $41 \%$ of responses have been obtained in Pts pre-treated with hormones. Eleven of them were refractory to TAM and our findings are in agreement with data from Buzzi et al (38) concerning the possibility of overcoming of resistance to the anti-estrogen with IFN. Using B-IFN in combination with TAM, 59\% of responses were obtained in Pts pre-treated with chemotherapy showing a stabilisation of disease with deteriorating markers.

Clinical response seems not to be related to the receptor status of Pts, even if the number of observations was not adequate to make a statement regarding receptor significance. It is well known that receptor content may change from primary tumor to metastatic disease, but it can not be excluded that receptor status underwent some variations over time due to the different treatments. It can be hypothesized that IFN, $\mathrm{R}$, or both, as shown in studies performed in vitro, determined a receptor enhancement, which could occur not only in those cells considered $\mathrm{ER}^{+}$but also in the $\mathrm{ER}^{-}$ones.

Our Pts had, from the start of biological therapy, a median PFS and OS of 43 and 47.9 months, respectively. Even if it is not correct to compare data from different patient populations, Pts with hormone responsive tumors, from the MD Anderson (1) series, had a median PFS and OS of 14.3 and 28.6 months, respectively.

The major toxicity of our regimen was hepatic and it was observed in eight Pts. Two of them, with negative hepatitis markers, could have had increased liver toxicity of retinyl palmitate by alcohol abuse. Both Pts were asymptomatic and had a mild elevation of liver enzymes. The observation that $15 \%$ of our Pts had some form of autoimmune disease indicates in the response we observed some kind of immune mechanism might be involved.

Even if the role played by the single agent is difficult to clarify, the combination of TAM, B-IFN and R seems to be an effective treatment modality for MBC. No previous data either pre-clinical or clinical exist concerning this three-drug combination. Nevertheless, our findings are in agreement with results by other authors concerning the effectiveness of 
the association of IFN and TAM in human cancer cells (9-11). Moreover, they are in accordance with a study by our group showing that 13-cis retinoic acid and B-IFN have a synergistic effect in terms of growth inhibition in a CG-5 estrogen-sensitive breast cancer cell line (8).

The similarity of retinoic acid receptors and steroid hormone receptors, which are involved in the mediation of antiestrogen action should be kept in mind. Eighteen percent of our Pts, with $\mathrm{ER}^{+}$tumors, developed a new cancer in the controlateral breast after a median time of 9 years. This supports the idea that $\mathrm{ER}^{+} \mathrm{MBC}$, even in the presence of a complete response can recur and therefore a life-long surveillance should be maintained with a contemporary therapy administration.

\section{References}

1. Rahman ZU, Frye DK, Smith TL, Asmar L, Theriault RL, Buzdar AU and Hortobagyi GN: Results and long term followup for 1581 patients with metastatic breast carcinoma treated with standard dose doxorubicin-containing chemotherapy: a reference. Cancer 85: 104-111, 1999.

2. Buzdar AU: Current status of endocrine treatment of carcinoma of the breast. Semin Surg Oncol 6: 77-82, 1990.

3. Nabholtz JM, Buzdar A, Pollak M, Harwin W, Burton G, Mangalik A, Steinberg M, Webster A and von Euler M: Anastrozole is superior to tamoxifen as first-line therapy for advanced breast cancer in postmenopausal women: results of a North American multicenter randomized trial. Arimidex Study Group. J Clin Oncol 18: 3758-3767, 2000.

4. Mouridsen H, Gershanovich M, Sun Y, Perez-Carrion R, Boni C, Monnier A, Apffelstaedt J, Smith R, Sleeboom HP, Jaenicke F, Pluzanska A, Dank M, Becquart D, Bapsy PP, Salminen E, Snyder R, Chaudri-Ross H, Lang R, Wyld P and Bhatnagar A: Phase III study of letrozole versus tamoxifen as first-line therapy of advanced breast cancer in postmenopausal women: analysis of survival and update of efficacy from the International Letrozole Breast Cancer Group. J Clin Oncol 21: 2101-2109, 2003.

5. Paridaens R, Dirix L, Lohrisch C, Beex L, Nooij M, Cameron D, Biganzoli L, Cufer T, Duchateau L, Hamilton A, Lobelle JP and Piccart M: European Organization for the Research and Treatment of Cancer (EORTC) - Investigational Drug Branch for Breast Cancer (IDBBC). Mature results of a randomized phase II multicenter study of exemestane versus tamoxifen as first-line hormone therapy for postmenopausal women with metastatic breast cancer. Ann Oncol 14: 1391-1398, 2003.

6. Clarke R, Brenner N, Katzenellenbogen BS, Thompson EW, Normal MJ, Koppi C, et al: Progression of human breast cancer cells from hormone-dependent to hormone-independent growth both in vitro and in vivo. Proc Natl Acad Sci USA 86: 3649-3653, 1989.

7. Recchia F, Sica G, de Filippis S, Discepoli S, Rea S, Torchio P and Frati L: Interferon-beta, retinoids, and tamoxifen in the treatment of metastatic breast cancer: a phase II study. J Interferon Cytokine Res 15: 605-610, 1995.

8. Lama G, Angelucci C, Recchia F and Sica G: Effect of 13-cisretinoic acid, tamoxifen and interferon on the growth of human breast cancer cells. Cancer Lett 100: 181-189,1996.

9. Lindner DJ and Borden EC: Synergistic antitumor effects of a combination of interferon and tamoxifen on estrogen receptorpositive and receptor-negative human tumor cell lines in vivo and in vitro. J Interferon Cytokine Res 17: 681-693, 1997.

10. Goldstein D, Bushmeyer SM, Witt PL, Jordan VC and Borden EC: Effect of type I and II interferons on cultured human breast cells: interaction with oestrogen receptors and with tamoxifen. Cancer Res 49: 2698-2702, 1989.

11. Porzsolt F, Otto AM, Traushel LB, Buck C, Wawer AW and Schonenberger $\mathrm{H}$ : Rationale for combining tamoxifen and interferon in the treatment of advanced breast cancer. J Cancer Res Clin Oncol 115: 465-469, 1989.

12. Van Den Berg HW, Lehaey WJ, Lynch M, Clarke R and Nelson J: Recombinant human interferon alpha increases oestrogen receptors in human breast cancer cells (ZR-75-1) and sensitises them to the anti-proliferative effect of tamoxifen. Br J Cancer 55: $255-257,1987$
13. Sica G, Natoli V, Stella G and Del Bianco S: Effect of natural beta-interferon on cell proliferation and steroid receptor level in human breast cancer cells. Cancer 60: 2419-2423, 1987.

14. Sporn MB and Roberts AB: Role of retinoids in differentiation and carcinogenesis. Cancer Res 43: 3034-3040, 1983.

15. Koga M and Sutherland R: Retinoic acid acts synergistically with 1,25-dihydroxy vitamin D3 or antioestrogen to inhibit T-47D human breast cancer cell proliferation. J Steroid Biochem Mol Biol 394: A455-A460, 1991

16. Butler WB and Fontana JA: Responses to retinoic acid of tamoxifen-sensitive and resistant sublines of human breast cancer cell line MCF-7. Cancer Res 1992: 6164-6167, 1992.

17. Clarke CL, Roman SD and Graham J: Progesterone receptor regulation by retinoic acid in the human breast cancer cell line T-47D. J Biol Chem 265: 12694-12700, 1990.

18. Mozaffari F, Lindemalm C, Choudhury A, GranstamBjörneklett H, Helander I, Lekander M, Mikaelsson E, Nilsson B, Ojutkangas ML, Osterborg A, Bergkvist L and Mellstedt $\mathrm{H}$ : NK-cell and T-cell functions in patients with breast cancer: effects of surgery and adjuvant chemo- and radiotherapy. Br J Cancer 97: 105-111, 2007.

19. Leon JA, Gutierrez MC, Jiang H, Estabrook A, Waxman S and Fisher PB: Modulation of the antigenic phenotype of human breast carcinoma cells by modifiers of protein kinase $\mathrm{C}$ activity and recombinant human interferons. Cancer Immunol Immunother 35: 315-324, 1992.

20. Prabhala RH, Garenwal HS, Hicks MJ, Sampliner RE and Watson RR: The effects of 13-cis-Retinoic acid and betacarotene on cellular immunity in humans. Cancer 67: 1556-1560, 1991.

21. Manni A and Arafah BM: Tamoxifen-induced remission in breast cancer by escalating the dose to $40 \mathrm{mg}$ daily after progression on $20 \mathrm{mg}$ daily: a case report and review of the literature. Cancer 48: 873-875, 1981 .

22. Mouridsen H, Palshof T, Patterson J and Battersby L: Tamoxifen in advanced breast cancer. Cancer Treat Rev 5: 131-141, 1978.

23. Simon R: Optimal two-stage designs for phase II clinical trials. Control Clin Trials 10: 1-10, 1989.

24. Kaplan EL and Meier P: Nonparametric estimation from incomplete observations. J Am Stat Assoc 53: 457-481, 1958

25. Colleoni M, Gelber S, Goldhirsch A, Aebi S, CastiglioneGertsch M, Price KN, Coates AS and Gelber RD: International Breast Cancer Study Group. Tamoxifen after adjuvant chemotherapy for premenopausal women with lymph node-positive breast cancer: International Breast Cancer Study Group Trial 13-93. J Clin Oncol 24: 1332-1341, 2006.

26. Gibson LJ, Dawson CK, Lawrence DH and Bliss JM: Aromatase inhibitors for treatment of advanced breast cancer in post-menopausal women. Cochrane Database Syst Rev 24: CD003370, 2007.

27. Pouillart P, Palangie T, Jouve M, Garcia-Giralt E, Fridman WH, Magdelenat $\mathrm{H}$, et al: Administration of fibroblast interferon to patients with advanced breast cancer: possible effect on skin metastasis and on hormone receptors. Eur J Cancer Clin Oncol 18: 929-935, 1982

28. Sica G, Iacopino F, Lama G, Amadori D, Baroni M, Lo Sardo F, Malacarne P, et al: Steroid receptor enhancement by natural B-interferon in advanced breast cancer. Eur J Cancer 29: 329-333, 1993.

29. Toma S, Isnardi L, Raffo P, Dastoli G, De Francisci E, Riccardi L, Palumbo R and Bollag W: Effects of all-trans-retinoic acid and 13-cis-retinoic acid on breast-cancer cell lines: growth inhibition and apoptosis induction. Int J Cancer 70: 619-627, 1997.

30. Patel JB, Mehta J, Belosay A, Sabnis G, Khandelwal A, Brodie AM, Soprano DR and Njar VC: Novel retinoic acid metabolism blocking agents have potent inhibitory activities on human breast cancer cells and tumour growth. Br J Cancer 96: 1204-1215, 2007

31. Belosay A, Brodie AM and Njar VC: Effects of novel retinoic acid metabolism blocking agent (VN/14-1) on letrozoleinsensitive breast cancer cells. Cancer Res 66: 11485-11493, 2006.

32. Lindner DJ, Borden EC and Kalvakolanu DV: Synergistic antitumor effects of a combination of interferons and retinoic acid on human tumor cells in vitro and in vivo. Clin Cancer Res 3: 931-937, 1997

33. Finke J, Ferrone S, Frey A, Mufson A and Ochoa A: Where have all the T cells gone? Mechanisms of immune evasion by tumors. Immunol Today 4: 158-160, 1999. 
34. Guadagni F, Schlom J, Johnston WW, Szpak CA, Goldstein D, Smalley R, Simpson JF, Borden EC, Pestka S and Greiner JW: Selective interferon-induced enhancement of tumor-associated antigens on a spectrum of freshly isolated human adenocarcinoma cells. J Natl Cancer Inst 81: 502-512, 1989.

35. Huang B, Pan PY, Li Q, Sato AI, Levy DE, Bromberg J, Divino CM, et al: $\mathrm{Gr}-1^{+} \mathrm{CD} 115^{+}$immature myeloid suppressor cells mediate the development of tumor-induced $\mathrm{T}$ regulatory cells and T-cell anergy in tumor-bearing host. Cancer Res 66: 1123-1131, 2006.

36. Hengesbach L and Hoag K: Physiological concentrations of retinoic acid favor myeloid dendritic cell development over granulocyte development in cultures of bone marrow cells from mice. J Nutr 134: 2653-2659, 2004.
37. Kusmartsev S, Cheng F, Yu B, Nefedova Y, Sotomayor E, Lush R, et al: All-trans-retinoic acid eliminates immature myeloid cells from tumor-bearing mice and improves the effect of vaccination. Cancer Res 63: 4441-4449, 2003.

38. Buzzi F, Brugia M, Rossi G, Giustini L, Scoponi C and Sica G: Combination of beta interferon and tamoxifen a new way to overcome clinical resistance to tamoxifen in advanced breast cancer. Anticancer Res 2: 869-872, 1992. 